\title{
Maternal interpersonal trauma and cord blood IgE levels in an inner-city cohort: $A$ life-course perspective
}

\author{
Michelle Judith Sternthal, PhD, ${ }^{a}$ Michelle Bosquet Enlow, PhD, ${ }^{\mathrm{b}}$ Sheldon Cohen, PhD, ${ }^{\mathrm{c}}$ Marina Jacobson Canner, MA, \\ John Staudenmayer, PhD, ${ }^{\mathbf{e}}$ Kathy Tsang, MHA, ${ }^{\mathbf{d}}$ and Rosalind J. Wright, MD, $\mathbf{M P H}^{\mathbf{a}, \mathbf{d}}$ Boston and Amherst, Mass, \\ and Pittsburgh, $\mathrm{Pa}$
}

Background: Prenatal stress affects immunocompetence in offspring, although the underlying mechanisms are not well understood.

Objective: We sought to examine associations between maternal lifetime interpersonal trauma (IPT) and cord blood total IgE levels in a sample of urban newborns $(n=478)$.

Methods: Maternal IPT during childhood and adolescence (birth to 17 years), adulthood (18 years to index pregnancy), and the index pregnancy were ascertained by using the Revised Conflict Tactics Scale at $28.4 \pm 7.9$ weeks' gestation. Cord blood IgE levels were derived by using a fluoroenzyme immunoassay. We examined effects of maternal IPT on increased cord blood IgE levels (upper quartile, $1.08 \mathrm{IU} / \mathrm{mL}$ ) by using logistic regression, adjusting for confounders and mediating variables.

Results: Maternal trauma was categorized as unexposed $(\mathbf{n}=$ $285[60 \%]$ ), early (childhood and/or teenage years only, $n=107$ [22\%]), late (adulthood and/or index pregnancy only, $n=29$ $[6 \%]$ ), and chronic (early and late, $n=57$ [12\%]) exposure. Relative to no IPT, early (odds ratio [OR], 1.78; 95\% CI, 1.053.00) and chronic maternal IPT (OR, 2.25; 95\% CI, 1.19-4.24) were independently associated with increased IgE levels in unadjusted analyses. When adjusting for standard controls, including maternal age and race, season of birth, child's sex, and childhood and current socioeconomic status, early effects became nonsignificant (OR, 1.48; 95\% CI, 0.85-2.58). Chronic exposure remained significant in fully adjusted models, including standard controls, current negative life events,

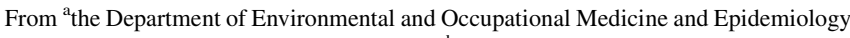
Harvard School of Public Health, Boston; 'the Department of Psychiatry, Children' Hospital Boston, and Harvard Medical School, Boston; ' ogy, Carnegie Mellon University, Pittsburgh; ${ }^{\mathrm{d}}$ the Channing Laboratory, Brigham \& Women's Hospital, Harvard Medical School, Boston; and ethe Department of Mathematics and Statistics, University of Massachusetts, Amherst.

The Asthma Coalition on Community, Environment, and Social Stress study was funded by grants R01ES10932, U01HL072494, and R01HL080674. M. S. is supported by grant T32-ES07069-29 and the Leaves of Grass Foundation.

Disclosure of potential conflict of interest: M. J. Sternthal and R. J. Wright have received research support from the National Institutes of Health and the Leaves of Grass Foundation. M. B. Enlow has received research support from the National Institute of Mental Health and the Children's Brain Research Foundation. S. Cohen has received research support from the National Institutes of Health and the MacArthur Foundation and consulted for Johnson \& Johnson Consumer and Personal Products Worldwide. The rest of the authors have declared that they have no conflict of interest.

Received for publication April 6, 2009; revised July 13, 2009; accepted for publication July 16, 2009.

Available online September 14, 2009.

Reprint requests: Michelle Judith Sternthal, $\mathrm{PhD}$, Department of Environmental and Occupational Medicine and Epidemiology, Harvard School of Public Health, 401 Park Dr, Boston, MA 02215. E-mail: msternth@hsph.harvard.edu.

0091-6749/\$36.00

(C) 2009 American Academy of Allergy, Asthma \& Immunology

doi:10.1016/j.jaci.2009.07.030 allergen exposure, and potential pathway variables (maternal atopy, prenatal smoking, and birth weight; OR, 2.18; 95\% CI, 1.06-4.50).

Conclusion: These data link chronic trauma over the mother's life course with increased IgE levels in infants at birth. Research examining associations between maternal trauma and indicators of offspring's atopic risk might be particularly relevant in innercity high-risk populations. (J Allergy Clin Immunol 2009;124:954-60.)

Key words: Interpersonal trauma, life course, pregnancy, cord blood IgE, urban asthma

Hypersensitivity to environmental stimuli associated with $\operatorname{IgE}$ production is a fundamental feature of atopy, predisposing individuals to clinical disorders, including allergic rhinitis, atopic dermatitis, and allergic asthma. ${ }^{1}$ Evidence linking psychologic stress to allergy and asthma ${ }^{2}$ suggests that the association is mediated through effects on neuroimmunoregulatory processes that set the stage for altered immune reactivity and enhanced IgE production. ${ }^{3}$ Examining relationships between stress exposure during critical developmental periods and biomarkers of immune response that precede clinical disease might elucidate pathways that increase vulnerability to atopy in response to stress.

Immune development and the predisposition to atopy begin during gestation. ${ }^{1,3}$ Increased cord blood IgE levels are an early indicator of immune polarization toward an atopic phenotype associated with the development of allergic diseases in later childhood. ${ }^{4}$ Prospective studies demonstrate that increased IgE levels detected during infancy track with age. ${ }^{5,6}$

Maternal stress influences fetal programming of physiologic systems (eg, immune function); consequently, stress effects might be transgenerational. ${ }^{3}$ Prenatal stress might disrupt maternal physiology (the hypothalamic-pituitary-adrenal [HPA] axis and the sympathetic-adrenal-medullary system), which, in turn, upregulates maternal and fetoplacental $\mathrm{T}_{\mathrm{H}} 2$ cytokine production. The enhanced $\mathrm{T}_{\mathrm{H}} 2$ cytokine/chemokine milieu might affect fetal immune function, including IgE isotype development. ${ }^{3}$ Thus infants exposed to maternal stress in utero might be more likely to express increased IgE levels at birth. Because interpersonal trauma (IPT) is more likely to result in lasting biobehavioral sequelae (eg, neurohormonal disruption $)^{7}$ and intergenerational effects compared with other stressors, ${ }^{8}$ maternal trauma might be a particularly potent inducer of immunomodulatory effects starting in utero. ${ }^{9}$ Moreover, such stressors disproportionally burden urban, lower-income US populations, ${ }^{10}$ who are also overly burdened by allergic disorders.

Although studies of maternal stress and infant outcomes typically examine events occurring during pregnancy, ${ }^{11}$ we consider IPT across the mother's life course. The life-course 

Abbreviations used
HPA: Hypothalamic-pituitary-adrenal
IPT: Interpersonal trauma
NLE: Negative life event
OR: Odds ratio
R-CTS: Revised Conflict Tactics Scale
SES: Socioeconomic status
WIC: Women, Infants, and Children

perspective posits that some stressors might influence health through 2 mechanisms, early programming and cumulative pathways, in addition to more immediate effects. ${ }^{12}$ Early programming can occur if exposures during sensitive developmental periods have lasting psychobiologic sequelae. Both early childhood and adolescence have been identified as sensitive periods susceptible to the effects of stress. ${ }^{3,13}$ Exposure to IPT in earlier life can generate disrupted physiologic stress responses even several years after the trauma. ${ }^{14,15}$ Thus maternal IPT might be linked to infant health through more latent effects (ie, lasting effects from abuse in childhood/adolescence), proximate effects (ie, trauma experienced in or around the pregnancy), and cumulative life-course effects (ie, allostatic load of accumulated traumas over the mother's life). ${ }^{15,16}$ No human studies have examined the influence of maternal trauma on the expression of atopic phenotypes at birth.

We investigated the relationship between maternal IPT experienced over her life course and cord blood total IgE levels, a biomarker of atopic risk at birth, in an urban population-based study. We hypothesized that infants born to mothers with chronic trauma exposure (ie, both early in life and more proximate to the pregnancy) would be at greatest risk of expressing increased IgE levels.

\section{METHODS \\ Participants}

Participants were from the Asthma Coalition on Community, Environment, and Social Stress project, a prospective cohort originally funded to recruit 500 pregnant women and their children to study the effects of prenatal maternal and early-life stress on urban childhood asthma risk, as described elsewhere. ${ }^{17}$ Briefly, English- or Spanish-speaking pregnant women who were at least 18 years old and receiving prenatal care at Brigham \& Women's Hospital, Boston Medical Center, 3 metropolitan Boston community health centers, and their associated Women, Infants, and Children (WIC) programs were recruited between August 2002 and January 2007. Research assistants approached women receiving prenatal care on selected clinic days, of whom $78.1 \%$ were eligible and agreed to enroll. Those who chose not to participate answered a screener questionnaire including race/ethnicity, education, and annual household income; no significant differences in these covariates emerged between the participants and those who declined. The study was approved by the human studies committees at the Brigham \& Women's Hospital and Boston Medical Center; written informed consent was obtained in the subject's primary language (English or Spanish). Cord blood IgE levels and complete data on maternal IPT were available for 478 dyads. Comparison of children with and without IgE measurement showed no differences based on sociodemographics or maternal trauma exposure.

\section{Measures and procedures}

Baseline questionnaire data were obtained at $28.4 \pm 7.9$ weeks' gestation.

IPT. Mothers completed the Revised Conflict Tactics Scale (R-CTS) short form, which has previously documented reliability and validity. ${ }^{18}$ IPT was assessed during childhood ( $\leq 11$ years of age), adolescence (12-17 years of age), adulthood before the current pregnancy, and during the pregnancy. For each life stage, exposure was assessed by using the same 6 items, asking mothers whether anyone had ever pushed, grabbed, or shoved them; kicked, bit, or punched them; hit them with something that hurt their body; choked or burned them; forced them to have sexual activities; or physically attacked them in some other way. For the childhood period, the stem to the question was modified to ask, "Did anyone at least 5 years older than you ever..." followed by the items. Mothers were classified as exposed in each life period if they answered yes to any of the items.

Life-course IPT was categorized as (1) unexposed, (2) early (during childhood or teenage years only), (3) late (during adulthood and/or the index pregnancy only), or (4) chronic (both early and late) exposure. Relatively small numbers reported abuse only in childhood $(\mathrm{n}=42)$ or adolescence $(\mathrm{n}=32)$, and $61 \%$ of women experiencing IPT in adolescence also reported earlier childhood exposure; these categories were collapsed. Pregnancy and adulthood periods were also combined given the small number reporting abuse during pregnancy $(\mathrm{n}=21)$ and the high covariance between the 2 periods (67\% experiencing IPT during pregnancy also reported exposure in adulthood before the pregnancy).

Maternal and cord blood IgE levels. Sera from mothers collected during pregnancy and venous placental cord blood collected at birth were analyzed with the CAP fluorescent enzyme immunoassay (Pharmacia [now Phadia], Uppsala, Sweden) for total IgE levels (in international units per milliliter). A modified protocol was used for cord blood, reducing the lower limit of detection from 2.0 to $0.2 \mathrm{IU} / \mathrm{mL} .{ }^{19}$ Maternal IgE levels, which were available for 320 mothers, were considered a control variable in secondary sensitivity analyses detailed below.

Covariates. All analyses were adjusted for the child's sex and season of birth, given documented associations with cord blood IgE levels. ${ }^{20}$ Potential confounders included younger maternal age, low socioeconomic status (SES), and minority status, which are associated with IPT and asthma/atopy in both mothers and infants. ${ }^{9}$ Thus standard controls included maternal age, race/ethnicity, and education. The mother's current SES was further controlled through a 3-item index of economic difficulties. ${ }^{21}$ Items were scored on a 5-point scale and summed; higher scores indicated greater difficulties (range, $0-15$ ). Lower childhood SES is linked to increased abuse risk ${ }^{22}$ and asthma risk in the mothers, ${ }^{23}$ and both can influence atopic susceptibility in the next generation. Each mother reported whether her parents owned their home during her childhood. A binary indicator of parental home ownership over the mother's childhood (ages 0-20 years) was included as a marker of childhood socioeconomic circumstances. ${ }^{24-26}$ Women experiencing IPT during pregnancy are more likely to report other adverse events. ${ }^{27}$ Using the 63 -item Crisis in Family Systems-Revised, which has been validated in English and Spanish, ${ }^{28,29}$ participants indicated whether they experienced events spanning several domains (community violence/safety and housing issues) during the pregnancy and rated endorsed events as positive, negative, or neutral. Items related to IPT were omitted because the R-CTS assessed these exposures. A summary score based on the total events rated negative was calculated (current negative life events [NLEs]). Lower-SES populations disproportionately exposed to IPT might also live in poor housing with increased exposure to atopy-exacerbating environmental factors (eg, indoor allergens ${ }^{30}$ ). Settled dust was collected during pregnancy from the mother's bed and bedroom floor by using a standardized protocol. ${ }^{31}$ Dust mite (Der $\mathrm{f} 1$ and Der $\mathrm{p} 1$ ) and cockroach (Bla g 1 and Bla g 2) allergens were measured with an mAb ELISA (Indoor Biotechnologies, Charlottesville, Va). Exposure was defined as previously described (ie, Der 1 and Der p 1, >0.20 $\mu \mathrm{g} / \mathrm{g}$; Bla g 1 and Bla g 2, >2 U/g). ${ }^{32}$

Finally, we assessed potential pathways through which maternal trauma history might contribute to newborn IgE expression. IPT is associated with risk factors previously correlated with offspring's atopy or cord blood IgE levels, including preterm delivery and low birth weight ${ }^{33,34}$ and smoking. ${ }^{35}$ Thus we adjusted for a continuous measure of birth weight for gestational age ${ }^{36}$ and for maternal prenatal smoking based on self-report using timeline follow-back procedures. ${ }^{37}$ Women exposed to IPT in earlier life might themselves be at increased risk of asthma/atopy, and maternal atopy predicts increased cord blood IgE levels. Maternal atopy was defined as a clinician-based lifetime diagnosis of asthma, hay fever, and/or eczema. 


\section{Statistical analysis}

All analyses used SAS statistical software version 9 (SAS Institute, Inc, Cary, NC). Cord blood and maternal IgE levels were log transformed because of skewness, assigning a value of 0.10 to undetectable values. The association between the mean of log cord blood IgE levels and trauma exposure categories was not significant. Cord blood levels were a priori divided into quartiles, with serum levels at or greater than the upper $25 \%(1.08 \mathrm{IU} / \mathrm{mL})$ considered high. This value approximates levels in prior studies demonstrating associations between more extremely increased cord blood IgE levels and increased atopic risk in later childhood. ${ }^{38-40}$ Categorization also makes the decision of the value to define the nondetectable values as irrelevant and can model a nonlinear or threshold relationship between IPT and IgE levels.

Relationships between IPT and covariates and between cord blood $\operatorname{IgE}$ levels and covariates were examined by using Kruskal-Wallis ANOVA to test differences in means for multiple groups and the Pearson $\chi^{2}$ statistic to test differences in proportions. Bonferroni tests and Wilcoxon rank sum testing were conducted for each 2-group pairing within multigroup comparisons. Logistic regressions predicted increased cord blood IgE levels. We first fit a univariate model including only IPT. We next added control variables, followed by NLEs and home allergen exposure. The final model included hypothesized pathway variables. Results are presented as odds ratios (ORs), with 95\% CIs in parentheses.

In secondary analysis with a reduced sample, we further adjusted for prenatal maternal total IgE levels. ${ }^{41}$ Analyses were also run with different IgE cutoff points to examine sensitivity to alternative specifications.

\section{RESULTS}

Tables I and II describe sample characteristics stratified by periods of IPT over the mother's life course and by IgE categories, respectively. Overall maternal age was $26.6 \pm 5.7$ years. The sample was largely Hispanic (59\%) or black (30\%, Table I). More respondents experienced no IPT than early $(60 \%$ vs $22 \%, P<.001)$, late $(60 \%$ vs $6 \%, P<.001)$, or chronic $(60 \%$ vs $12 \%, P<0.001)$ IPT. More Hispanics relative to blacks or other/mixed race subjects reported no IPT $(66 \%$ vs $52 \%[P<.003]$ and $49 \%[P<.03]$, respectively); a higher proportion of blacks reported early-life IPT than Hispanics $(31 \%$ vs $17 \%, P<.001)$. More atopic mothers reported chronic abuse than nonatopic mothers $(16.5 \%$ vs $9 \%$, $P<.02)$. Mothers with chronic IPT reported more frequent NLEs compared with unexposed mothers $(4.2 \pm 3.92$ vs $1.7 \pm 2.3$, $P<.001)$, those with early exposure $(4.2 \pm 3.92$ vs $2.8 \pm 2.7$, $P<.001)$, and those with later-life exposure only $(4.2 \pm 3.92 \mathrm{vs}$ $2.1 \pm 2.4, P<.001)$. Current economic difficulties were higher for late-life exposure compared with no exposure $(6.4 \pm 2.8$ vs $5.4 \pm 2.3, P<.03)$, chronic versus no exposure $(6.7 \pm 2.7$ vs 5.4 $\pm 2.3, P<.001)$, and chronic compared with early exposure $(6.7$ \pm 2.7 vs $5.8 \pm 2.6, P<.03)$. No significant differences emerged across IPT groups based on parental home ownership, maternal education, child's sex, birth weight, maternal age, household allergen exposure, or season of birth. Prenatal smoking prevalence for mothers reporting chronic IPT $(23 \%)$ was more than double that of mothers reporting no exposure (11\%), early IPT $(11 \%)$, and late IPT (10\%), although these differences were not significant.

More blacks than Hispanics had increased cord blood IgE levels (n $=45[32 \%]$ vs $\mathrm{n}=58$ [21\%], $P<.01)$, as did offspring of atopic versus nonatopic mothers $(\mathrm{n}=55[32 \%]$ vs $\mathrm{n}=65[21 \%], P<.00)$ and infants whose mothers reported their family not owning their home during her childhood versus those reporting parental home ownership ( $\mathrm{n}=61$ [35\%] vs $\mathrm{n}=45$ [18\%], $P<.01$; Table II).

No significant differences emerged between trauma exposure groups in terms of $\operatorname{IgE}$ geometric means $(P=.28$, Table III). However, among the women experiencing IPT during any life period, there was increased prevalence of more extreme levels of cord blood $\mathrm{IgE}$ (indexed as $\geq 1.08 \mathrm{IU} / \mathrm{mL}$; early life, $30 \%$ $[P<.03]$, late life, 35\% $[P<.04]$, chronic IPT, 35\% $[P<.01]$; all relative to no IPT, $20 \%$ ).

The ORs (95\% CIs) of increased cord blood IgE levels by mother's IPT exposure categories, when considered simultaneously, are presented in Table IV. Early IPT (OR, 1.78; 95\% CI, 1.05-3.00) and chronic exposure (early and late; OR, 2.25; $95 \%$ CI, 1.19-4.24) were independently associated with increased IgE levels in unadjusted analysis; later-life trauma was borderline significant. Adjusting for sociodemographics and season of birth (model 1) attenuated the effects for early IPT to nonsignificance. Stepwise analyses adding in sociodemographic indicators one at a time and in pairs (not shown) revealed that race/ethnicity and home ownership (OR for early IPT, 1.51; 95\% CI, 0.88-2.60) accounted for the attenuation of early IPT effects. The effect estimate for chronic exposure to IPT was not changed in magnitude or precision (ie, confidence bounds were unchanged), even with inclusion of posited pathway variables (ie, birth weight for gestational age, maternal atopy, and prenatal smoking).

When adjusting for all covariates plus maternal IgE levels, the association between maternal IPT and cord blood IgE levels remained (OR for early IPT, 1.43 [95\% CI, 0.66-3.10]; OR for late IPT, 3.26 [95\% CI, 1.07-9.96]; and OR for chronic IPT, 2.92 [95\% CI, 1.22-6.96). There was also a significant independent effect of maternal IgE level (per 1-unit increase in $\log$ maternal IgE level; OR, 1.72; 95\% CI, 1.35-2.18). We also considered alternative IgE cutoffs for the top $20 \%$ and $33 \%$ to examine sensitivity to alternative specifications. When a more extreme (top 20\%) cutoff for high IgE level was used $(1.40 \mathrm{IU} / \mathrm{mL})$, the effects increased [OR for early IPT, 1.80 [95\% CI, 0.98-3.31]; OR for late IPT, 2.47 [95\% CI, 0.936.51]; and OR for chronic IPT, 2.25 [95\% CI, 1.03-4.91]) in fully adjusted models. When using a cutoff below levels previously linked with atopic clinical disorders in children (ie, upper tertile for $\operatorname{IgE}[0.79 \mathrm{IU} / \mathrm{mL}]$ ), there was no longer significance (ORs were 1.35 [95\% CI, 0.80-2.29], 1.86 [95\% CI, 0.79-4.39], and 1.51 [95\% CI, 0.76-3.02] for early, late, and chronic IPT, respectively).

\section{DISCUSSION}

These analyses provide the first suggestion that chronic exposure to IPT across the mother's life course is associated with increased IgE levels in newborns when adjusted for race/ethnicity, childhood and current SES, other current NLEs, household allergens, season of birth, and potential mediators (eg, prenatal smoking, birth weight for gestational age, maternal atopy and $\operatorname{IgE}$ level). Although results are most consistent with cumulative lifecourse effects in the mother potentiating the child's immune response toward $\mathrm{IgE}$ production, there is some support for independent effects of exposure during early life, when these mothers might be more vulnerable to lasting programming effects of stress (childhood and adolescence). ${ }^{3,13}$ Finally, our analyses did not suggest that birth weight for gestational age, maternal smoking, or maternal atopy operated as mediators.

In general, physiologic systems operate at higher or lower levels than in normal homeostasis in response to stress. Immune and neural defensive biologic responses important for short-term reaction to stress might produce long-term damage if not checked and terminated. Such chronic stress effects have been conceptualized as "allostatic load." Accumulated experiences of IPT across the mother's life might result in such accommodation with 
TABLE I. Distribution of covariates across IPT exposure categories over the mother's life course $(n=478)$

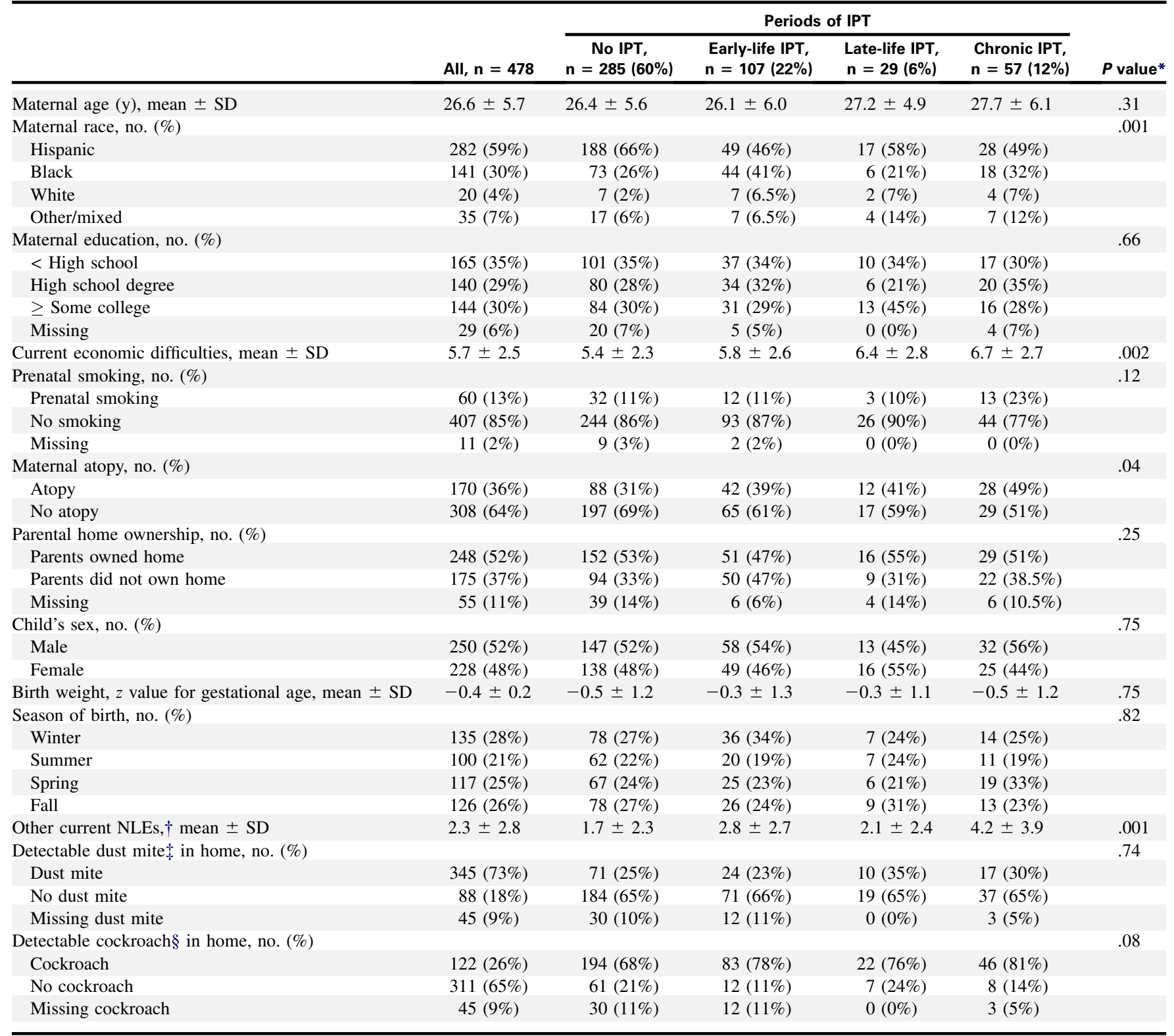

*For differences between nonmissing multigroup comparisons using ANOVA and $\chi^{2}$ distribution.

†Current NLEs other than IPT.

¿Detectable levels for dust mites (Der f 1 and Der $\mathrm{p} 1,>0.02 \mu \mathrm{g} / \mathrm{g}$ ).

$\S$ Detectable levels for cockroach allergens (Bla g 1, >0.40 U/g; Bla g 2, >1.0 U/g).

disturbed regulation of the maternal stress systems (eg, HPA axis and sympathetic-adrenal-medullary system), which might, during pregnancy, influence fetal immune development. ${ }^{8,42}$ The fetal HPA axis can be stimulated to amplify fetal glucocorticoid excess, as well as to activate additional elements of the fetal stress response (ie, catecholamines) affecting the developing immune system. Alterations in stress-induced maternal cortisol levels might also influence $\mathrm{T}_{\mathrm{H}} 2$ cell predominance, perhaps through its influence on cytokine production. ${ }^{3}$ This might induce an enhanced shift toward $\mathrm{T}_{\mathrm{H}}$ 2-mediated humoral immunity and $\operatorname{IgE}$ production. Although these in utero responses might be adaptive in the short-term and geared toward coping with anticipated environmental challenges, ultimately they might exact a toll, contributing to increased risk of atopic diseases in later life.
Effects of childhood adversity might operate directly through latent programming effects or more indirectly through an increased likelihood of adult NLE exposure, as well as enhanced life-stress sensitivity, the so-called stress sensitization model. ${ }^{43}$ In models adjusting for later-life exposure, as well as chronic exposure, the mother's early-life experience of IPT was significantly associated with increased cord blood IgE levels. That this attenuated when adding race/ethnicity, childhood SES, or both is interesting. We saw in the descriptive analysis that blacks were more likely to report early-life abuse, which might have contributed to the attenuation. Higher childhood SES has been associated with more positive caregiving and less family chaos, which might buffer the adverse effects of early childhood adversity. In accordance with this hypothesis, recent analyses conducted in the Fragile Families and Child 
TABLE II. Distribution of covariates across IgE categories $(n=478)$

\begin{tabular}{|c|c|c|c|}
\hline & \multicolumn{2}{|c|}{ IgE level categories } & \multirow[b]{2}{*}{$P$ value* } \\
\hline & $\begin{array}{c}\text { Low, } \\
n=358 \\
(75 \%)\end{array}$ & $\begin{array}{c}\text { High, } \\
\mathrm{n}=120 \\
(25 \%)\end{array}$ & \\
\hline Maternal age $(y)$, mean \pm SD & $27 \pm 5.7$ & $26 \pm 5.7$ & .03 \\
\hline Maternal race, no. (\%) & & & .05 \\
\hline Hispanic & $224(62 \%)$ & $58(48 \%)$ & \\
\hline Black & $96(27 \%)$ & $45(38 \%)$ & \\
\hline White & $13(4 \%)$ & $7(6 \%)$ & \\
\hline Other/mixed & $25(7 \%)$ & $10(8 \%)$ & \\
\hline Maternal education, no. (\%) & & & .23 \\
\hline$<$ High school & $131(37 \%)$ & $34(28 \%)$ & \\
\hline High school degree & $100(28 \%)$ & $40(33 \%)$ & \\
\hline$\geq$ Some college & $105(29 \%)$ & $39(33 \%)$ & \\
\hline Missing & $22(6 \%)$ & $7(6 \%)$ & \\
\hline $\begin{array}{l}\text { Current economic difficulties, } \\
\text { mean } \pm \mathrm{SD}\end{array}$ & $5.6 \pm 2.4$ & $6.1 \pm 2.7$ & .04 \\
\hline Prenatal smoking, no. (\%) & & & .12 \\
\hline Prenatal smoking & $46(13 \%)$ & $14(12 \%)$ & \\
\hline No smoking & $304(85 \%)$ & $103(86 \%)$ & \\
\hline Missing & $8(2 \%)$ & $3(2 \%)$ & \\
\hline Maternal atopy, no. (\%) & & & .01 \\
\hline Atopy & $115(32 \%)$ & $55(46 \%)$ & \\
\hline No atopy & $243(68 \%)$ & $65(54 \%)$ & \\
\hline Parental home ownership, no. (\%) & & & .001 \\
\hline Parents owned home & $203(57 \%)$ & $45(37 \%)$ & \\
\hline Parents did not own home & $114(32 \%)$ & $61(51 \%)$ & \\
\hline Missing & $41(11 \%)$ & $14(12 \%)$ & \\
\hline Child's sex, no. (\%) & & & .49 \\
\hline Male & $184(51 \%)$ & $66(55 \%)$ & \\
\hline Female & $174(49 \%)$ & $54(45 \%)$ & \\
\hline $\begin{array}{l}\text { Birth weight, } z \text { value for } \\
\text { gestational age, mean } \pm \text { SD }\end{array}$ & $-0.4 \pm 1.2$ & $-0.4 \pm 1.3$ & .72 \\
\hline Season of birth, no. (\%) & & & .45 \\
\hline Winter & $95(27 \%)$ & $40(33 \%)$ & \\
\hline Summer & $79(22 \%)$ & $21(17 \%)$ & \\
\hline Spring & $90(25 \%)$ & $27(23 \%)$ & \\
\hline Fall & $94(26 \%)$ & $32(27 \%)$ & \\
\hline Other Current NLEs, $\uparrow$ mean \pm SD & $2.2 \pm 2.6$ & $2.5 \pm 3.1$ & .35 \\
\hline Detectable dust mitef in home, no. (\%) & & & .97 \\
\hline Dust mite & $235(66 \%)$ & $76(63 \%)$ & \\
\hline No dust mite & $92(26 \%)$ & $30(25 \%)$ & \\
\hline Missing dust mite & $31(8 \%)$ & $14(12 \%)$ & \\
\hline Detectable cockroach $\S$ in home, no. (\%) & & & .12 \\
\hline Cockroach & $72(20 \%)$ & $16(13 \%)$ & \\
\hline No cockroach & $255(71 \%)$ & $90(75 \%)$ & \\
\hline Missing cockroach & $31(9 \%)$ & $14(12 \%)$ & \\
\hline
\end{tabular}

*For differences between nonmissing multigroup comparisons using ANOVA and $\chi^{2}$ distribution.

†Current NLEs other than IPT.

† Detectable levels for dust mites (Der f 1 and Der p 1, >0.02 $\mu \mathrm{g} / \mathrm{g}$ ).

$\S$ Detectable levels for cockroach allergens (Bla g 1, >0.40 U/g; Bla g 2, $>1.0 \mathrm{U} / \mathrm{g}$ ).

Wellbeing Study $(\mathrm{n}=2117)$ demonstrated an association between maternal report of chronic domestic violence and an increased risk of physician-diagnosed asthma in children by age 3 years, with effects attenuated when mothers maintained positive caregiving in the context of violence. $^{44}$

These data suggest a nonlinear or threshold effect in that among women who had experienced IPT, the prevalence of more extreme increased levels of $\operatorname{IgE}$ in their infants at birth increased. Also, sensitivity analysis demonstrated that results were strongest when considering more extreme cutoff values for increased cord blood
IgE $(\geq 1.08$ and $\geq 1.40 \mathrm{IU} / \mathrm{mL})$. The more extreme levels might be most relevant as a preclinical marker of atopic disorders that develop in later childhood. Although there is no agreement on the threshold level that is associated with increased risk of clinical atopic disorders, several studies have found that cord blood $\operatorname{IgE}$ levels between 0.9 and $1.3 \mathrm{IU} / \mathrm{mL}$ are associated with significantly increased risk, particularly in relation to early sensitization in later childhood. ${ }^{38,45}$ Indeed, cord blood IgE levels in general are a weaker predictor of later atopic risk unless the more extreme levels are considered. Use of lower cutoff values in prior research ${ }^{46}$ results in significant changes in the sensitivity, specificity, and positive predictive value. Moreover, although population studies suggest that most children with atopic disease do not have increased IgE levels as newborns, those with especially increased $\operatorname{IgE}$ responsiveness are particularly predisposed to allergic disorders.

\section{Strengths and limitations}

The strengths of the current study include the assessment of trauma in different life periods using identical items, adjustment for a range of confounders across the life course and potential mediators, and the focus on a population at high risk for both trauma and atopy. Nevertheless, limitations should be noted.

First, all participants were recruited from specific prenatal clinics or affiliated WIC sites. These mothers might differ systematically from those not receiving prenatal care at these centers or participating in WIC, limiting generalizability.

Second, because measures of IPT are self-reported and retrospective, the potential for recall bias or social desirability response bias remains. However, any reporting bias would likely be in the direction of underreporting, biasing our results toward the null. Furthermore, the R-CTS is generally reliable because it asks about discrete objective events rather than global estimates of family interaction, which are less prone to inaccurate recall. ${ }^{47,48}$ Subjects might also inaccurately recall when past events have occurred. Although a blurring together of repeated events over childhood is possible, this is less likely to occur when subjects are asked to report for an age range ( $\leq 11$ years or $12-17$ years of age) rather than a specific age for each event. ${ }^{47}$

Third, although our sensitivity analyses suggest a threshold relationship between maternal lifetime IPT and cord blood IgE levels, we acknowledge that a dose-response relationship could be more rigorously explored by using a continuous measure of IPT. Although the R-CTS ascertains information on discrete types of violence, it does not capture other salient contextual features indicating violence severity (family/relationship context [ie, relationship of the perpetrator to the victim], injury severity, and abuse frequency). The lack of such salient information might have reduced the predictive power of the maternal IPT variables.

Finally, these analyses did not include psychologic or physiologic correlates of chronic prenatal stress, which future work should examine to test more directly the posited underlying mechanisms.

These analyses provide the first suggestion that maternal experiences of IPT might have transgenerational implications for asthma and allergy risk. The findings underscore the need to consider maternal stress beyond that experienced only during pregnancy when exploring intergenerational effects. Future studies linking maternal stress to chronic atopic diseases should consider a life-course approach. ${ }^{49}$ Further study of associations 
TABLE III. Distribution of cord blood IgE and geometric mean of IgE levels by IPT exposure

\begin{tabular}{|c|c|c|c|c|c|c|}
\hline \multirow[b]{3}{*}{ Periods of IPT } & \multicolumn{6}{|c|}{ Cord blood IgE level } \\
\hline & \multirow{2}{*}{$\begin{array}{c}\% \text { Nondetectable (LLOD } \\
<0.20)\end{array}$} & \multicolumn{4}{|c|}{ Percentiles } & \multirow[b]{2}{*}{ Geometric mean* (Cl) } \\
\hline & & $25 \%$ & $50 \%$ & $75 \%$ & Maximal & \\
\hline All & 24.7 & 0.20 & 0.51 & 1.08 & 100 & $0.50(0.45-0.56)$ \\
\hline No exposure & 24.2 & 0.20 & 0.46 & 0.91 & 46.3 & $0.46(0.40-0.53)$ \\
\hline Early-life IPT & 23.4 & 0.21 & 0.54 & 1.55 & 14.9 & $0.57(0.44-0.73)$ \\
\hline Late-life IPT & 27.6 & 0.14 & 0.51 & 1.65 & 3.6 & $0.49(0.30-0.80)$ \\
\hline
\end{tabular}

$L L O D$, Lower limit of detection.

TABLE IV. Maternal IPT exposure and cord blood total IgE levels: Logistic regression analyses

\begin{tabular}{|c|c|c|c|c|c|}
\hline \multirow[b]{3}{*}{ Predictors } & \multicolumn{5}{|c|}{ Cord blood $(n=478)$} \\
\hline & \multirow[b]{2}{*}{ Unadjusted models } & \multicolumn{4}{|c|}{ Multivariate models } \\
\hline & & Model 1* & Model 2† & Model $3 \ddagger$ & Model 4§ \\
\hline No IPT & - & - & - & - & - \\
\hline Childhood/ teen only & $1.78 \Phi(1.05-3.00)$ & $1.48(0.85-2.58)$ & $1.52(0.86-2.66)$ & $1.45(0.82-2.55)$ & $1.43(0.81-2.52)$ \\
\hline Adult/pregnancy only & $2.23 \|(0.97-5.13)$ & $2.19 \|(0.90-5.33)$ & $2.22 \|(0.91-5.43)$ & $2.33 \|(0.95-5.70)$ & $2.19 \|(0.89-5.38)$ \\
\hline
\end{tabular}

Results are presented as ORs and $95 \%$ CIs.

* Model 1 adjusts for maternal age, child's sex, maternal race, maternal education, current economic difficulties, season of birth, and parental home ownership.

† Model 2 adjusts for maternal age, child's sex, maternal race, maternal education, current economic difficulties, parental home ownership, season of birth, and other current NLEs

$\$$ Model 3 adjusts for maternal age, child’s sex, maternal race, maternal education, current economic difficulties, parental home ownership, season of birth, other current NLEs, and household allergens.

$\S$ Model 4 adjusts for maternal age, child's sex, maternal race, maternal education, current economic difficulties, parental home ownership, other current NLEs, household allergens, season of birth, birth weight for gestational age, maternal atopy, and maternal smoking during pregnancy.

$\| P<.10$.

$\Phi P<.05$.

between maternal IPT and child atopic risk should incorporate methodologies that minimize recall bias and assess IPT severity. Because interpersonal violence disproportionately affects members of racial/ethnic minorities and those of lower SES, such exposures might in part explain the excess burden of atopic disorders in these populations and warrants further research. ${ }^{9}$ Continued follow-up of this prospective pregnancy cohort will reveal whether lifetime maternal IPT influences the expression of allergic sensitization and asthma expression in these children.

Clinical implications: Studies examining prenatal stress and childhood atopy risk should also consider stress before pregnancy by taking a life-course perspective. Maternal chronic life stress, particularly IPT, might influence the child's developing immune system.

\section{REFERENCES}

1. Peden DB. Development of atopy and asthma: candidate environmental influences and important periods of exposure. Environ Health Perspect 2000;108(suppl 3): 475-82.

2. Chida YY, Hamer MM, Steptoe AA. A bidirectional relationship between psychosocial factors and atopic disorders: a systematic review and meta-analysis. Psychosom Med 2008;70:102-16.

3. Wright RJ. Prenatal maternal stress and early caregiving experiences: implications for childhood asthma risk. Paediatr Perinatol Epidemiol 2007;21:8-14.

4. Halken S. Early sensitisation and development of allergic airway disease-risk factors and predictors. Paediatr Respir Rev 2003;4:128-34.

5. Martinez FD, Wright AL, Taussig LM, Holberg CJ, Halonen M, Morgan WJ Asthma and wheezing in the first six years of life. N Engl J Med 1995;332:1330-8.
6. Sherrill DL, Stein R, Halonen M, Holberg CJ, Wright A, Martinez FD. Total serum $\mathrm{IgE}$ and its association with asthma symptoms and allergic sensitization among children. J Allergy Clin Immunol 1999;104:28-36.

7. Ford JD, Stockton P, Kaltman S, Green BL. Disorders of extreme stress (DESNOS) symptoms are associated with type and severity of interpersonal trauma exposure in a sample of healthy young women. J Interpers Violence 2006;21: 1399-416.

8. Yehuda R, Bierer LM. Transgenerational transmission of cortisol and PTSD risk Prog Brain Res 2008;167:121-35.

9. Wright RJ, Bosquet Enlow M. Maternal stress and perinatal programming in the expression of atopy. Exp Rev Clin Immunol 2008;4:535-8

10. Breslau N, Kessler RC, Chilcoat HD, Schultz LR, Davis GC, Andreski P. Trauma and posttraumatic stress disorder in the community the 1996 Detroit area survey of trauma. Arch Gen Psychiatry 1998;55:626-32.

11. Beydoun H, Saftlas AF. Physical and mental health outcomes of prenatal maternal stress in human and animal studies: a review of recent evidence. Paediatr Perinatol Epidemiol 2008;22:438-66.

12. Power C, Hertzman C. Social and biological pathways linking early life and adult disease. Br Med Bull 1997;53:210-21

13. Spear LP. The adolescent brain and age-related behavioral manifestations. Neurosci Biobehav Rev 2000;24:417-63.

14. Anda RF, Felitti VJ, Bremner JD, Walker JD, Whitfield C, Perry BD, et al. The enduring effects of abuse and related adverse experiences in childhood. Eur Arch Psychiatry Clin Neurosci 2006;256:174-86.

15. Rick S, Douglas DH. Neurobiological effects of childhood abuse. J Psychosoc Nurs Ment Health Serv 2007;45:47-54.

16. Holland P, Berney L, Blane D, Davey Smith G, Gunnell DJ, Montgomery SM. Life course accumulation of disadvantage: childhood health and hazard exposure during adulthood. Soc Sci Med 2000;50:1285-95.

17. Wright RJ, Franco S, Levy JI, Fortun K, Shields A, Subramanian SV, et al. Transdiscplinary research strategies for understanding socially patterned disease: the ACCESS project as a case study. Ciencia Saude Coletiva 2008;43:55-63.

18. Straus MA, Douglas EM. A short form of the Revised Conflict Tactics scales, an typologies for severity and mutuality. Violence Vict 2004;19:507-20. 
19. Platts-Mills TAE, Erwin EA, Allison AB, Blumenthal K, Barr M. The relevance of maternal immune responses to inhalant allergens to maternal symptoms, passive transfer to the infant, and development of antibodies in the first 2 years of life J Allergy Clin Immunol 2003;111:123-30.

20. Nilsson L, Bjorksten B, Hattevig G, Kjellman B, Sigurs N, Kjellman NIM. Season of birth as predictor of atopic manifestations. Arch Dis Child 1997;76:341-4.

21. Braveman PA, Cubbin C, Egerter S, Chideya S, Marchi KS, Metzler M, et al. Socioeconomic status in health research one size does not fit all. JAMA 2005;294:2879-88.

22. Brown J, Cohen P, Johnson JG, Salzinger S. A longitudinal analysis of risk factors for child maltreatment: findings of a 17-year prospective study of officially recorded and self-reported child abuse and neglect. Child Abuse Negl 1998;22:1065-78.

23. Jackson B, Kubzansky LD, Cohen S, Weiss S, Wright RJ, CARDIA Study. A matter of life and breath: childhood socioeconomic status is related to young adult pulmonary function in the CARDIA study. International Journal of Epidemiology 2004:33:271-8

24. Cohen S, Janicki-Deverts D. Childhood socioeconomic status and adult health. In: Adler N, Stewart J, editors. The biology of disadvantage: socioeconomic status and health. Annals of the New York Academy of Sciences. In press.

25. Cohen S, Doyle WJ, Turner RB, Alper CM, Skoner DP. Childhood socioeconomic status and host resistance to infectious illness in adulthood. Psychosom Med 2004; 66:553-8.

26. Miller G, Chen E. Unfavorable socioeconomic conditions in early life presage expression of proinflammatory phenotype in adolescence. Psychosom Med 2007;69:402-9.

27. Braveman P, Marchi K, Egerter S, Kim S, Metzler M, Stancil T, et al. Poverty, near-poverty, and hardship around the time of pregnancy. Matern Child Health J 2008 [Epub ahead of print].

28. Shalowitz MU, Berry CA, Rasinski KA, Dannhausen-Brun CA. A new measure of contemporary life stress: development, validation, and reliability of the CRISYS. Health Serv Res 1998;33:1381-402.

29. Berry CA, Quinn KA, Portillo N, Shalowitz MU. Reliability and validity of the Spanish version of the Crisis in Family Systems-Revised. Psychol Rep 2006;98: 123-32.

30. Rauh VA, Chew GR, Garfinkel RS. Deteriorated housing contributes to high cockroach allergen levels in inner-city households. Environ Health Perspect 2002; 110(suppl 2):323-7.

31. Chew GL. Monthly measurements of indoor allergens and the influence of housing type in a northeastern US city. Allergy 1999;54:1058.

32. Peters JL, Suglia SF, Platts-Mills TAE, Hosen J, Gold DR, Wright RJ. Relationships among prenatal aeroallergen exposure and maternal and cord blood IgE: Project ACCESS. J Allergy Clin Immunol 2009;123:1041-6.

33. Pekkanen J, Xu B, Jarvelin MR. Gestational age and occurrence of atopy at age 31: a prospective birth cohort study in Finland. Clin Exp Allergy 2001;31:95-102.

34. Neggers Y, Goldenberg R, Cliver S, Hauth J. Effects of domestic violence on preterm birth and low birth weight. Acta Obstet Gynecol Scand 2004;83:455-60.
35. Jun HJ, Rich-Edwards JW, Boynton-Jarrett R, Wright RJ. Intimate partner violence and cigarette smoking: association between smoking risk and psychological abuse with and without co-occurrence of physical and sexual abuse. Am J Public Health 2008;98:527-35.

36. Oken E, Kleinman KP, Rich-Edwards J, Gillman MQ. A nearly continuous measure of birth weight for gestational age using a United States national reference. BMC Pediatr 2003;3:6.

37. Brown RA, Burgess ES, Sales SD, Whiteley JA, Evans DM, Miller IW. Reliability and validity of a smoking timeline follow-back interview. Psychol Addict Behav 1998;12:101-12.

38. Halonen M, Stern D, Taussig LM, Wright A, George Ray C, Martinez FD. The predictive relationship between serum IgE levels at birth and subsequent incidences of lower respiratory illnesses and eczema in infants. Am Rev Respir Dis 1992;146: 866-70.

39. Edenharter G, Bergmann RL, Bergmann KE, Wahn V, Forster J, Zepp F, et al. Cord blood-IgE as risk factor and predictor for atopic diseases. Clin Exp Allergy 1998; 28:671-8.

40. Hansen LG, Halken S, Host A, Moller K, Osterballe O. Prediction of allergy from family history and cord blood IgE levels. A follow-up at the age of 5 years. Pediatr Allergy Immunol 1993;4:34-40.

41. Scirica CV, Gold DR, Ryan L, Abulkerim H, Celedón JC, Platts-Mills TAE, et al Predictors of cord blood IgE levels in children at risk for asthma and atopy. J Allergy Clin Immunol 2007;119:81-8.

42. de Weerth C, Buitelaar JK. Physiological stress reactivity in human pregnancy-a review. Neurosci Biobehav Rev 2005;29:295-312.

43. Monroe SM, Harkness KL. Life stress, the "Kindling" hypothesis, and the recurrence of depression: considerations from a life stress perspective. Psychol Rev 2005; 112:417-44

44. Suglia SF, Bosquet Enlow M, Kullowatz A, Wright RJ. Maternal intimate partner violence predicts increased asthma incidence in children: Buffering effects of supportive caregiving. Arch Pediatr Adolesc Med 2009;163:244-50.

45. Varonier HS, Lacourt GC, Assimacopoulos A. Cord serum IgE and early detection of the atopic phenotype: suitable for routine screening? Eur J Pediatr 1991;150: 844-6.

46. Cantani A. Neonatal immunology: the neonate at risk of atopy. In: Heilmann U, ed. Pediatric allergy, asthma and immunology. Heidelberg, Germany: Springer; 2008. 269-284.

47. Brewin CR, Andrews B, Gotlib IH. Psychopathology and early experience: a reappraisal of retrospective reports. Psychol Bull 1993;113:82-98.

48. Kessler RC, Magee WJ. Childhood family violence and adult recurrent depression. J Health Soc Behav 1994;35:13-27.

49. Ben-Shlomo Y, Kuh D. A life course approach to chronic disease epidemiology: conceptual models, empirical challenges and interdisciplinary perspectives. Int $\mathbf{J}$ Epidemiol 2002;31:285-93 